\title{
ON CERTAIN EXTREMAL PROBLEMS FOR FUNCTIONS WITH POSITIVE REAL PART
}

\author{
STEPHAN RUSCHEWEYH AND VIKRAMADITYA SINGH
}

\begin{abstract}
For the class $P$ of analytic functions $p(z), p(0)=1$, with positive real part in $|z|<1$, a type of extremal problems is determined which can be solved already within the set $p(z)=(1+\varepsilon z) /(1-\varepsilon z),|\varepsilon|=1$. One problem of this kind is to find the largest number $\rho(s, \mu)$ such that

$$
\operatorname{Re}\left\{p(z)+s z p^{\prime}(z) /(p(z)+\mu)\right\}>0,
$$

$|z| \leqslant \rho(s, \mu)$, for all $p \in P,-1 \neq \mu \in \mathbf{C}, s>0$. Sharp upper bounds for two other functionals over $P$ are also given.
\end{abstract}

1. Introduction. Let $P$ be the class of analytic functions $p(z)$ in the unit disc $\Delta=\{z|| z \mid<1\}$ with $p(0)=1, \operatorname{Re} p(z)>0$ in $\Delta$. Robertson [2], [3] and Sakaguchi [6] obtained variational formulae for $P$ and showed that, for fixed $z \in \Delta$, the extreme values of

$$
\operatorname{Re} F\left(p(z), z p^{\prime}(z)\right), \quad p \in P,
$$

where $F(u, v)$ is analytic in $(u, v) \in \mathbf{C}^{2}, \operatorname{Re} u>0$, are already attained by the functions

$$
\lambda\left(\frac{1+\varepsilon z}{1-\varepsilon z}\right)+(1-\lambda)\left(\frac{1+\bar{\varepsilon} z}{1-\bar{\varepsilon} z}\right), \quad \lambda \in[0,1],|\varepsilon|=1 .
$$

Zmorovič [10], [11] and others used this result to solve extremal problems in $P$. In many cases of interest, however, even this reduced problem is difficult to solve or appears insurmountable.

In the first part of this paper we present a theorem which characterises a class of extremal problems in $P$ which can be solved in

$$
P_{0}:=\{p \in P|p(z)=(1+\varepsilon z) /(1-\varepsilon z),| \varepsilon \mid=1\} .
$$

Our theorem rests on recent results of Suffridge [8] concerning the Hadamard product of starlike functions of order $\alpha$ (compare also Ruscheweyh and Sheil-Small [5] and Ruscheweyh [4]).

One problem which can be treated by our method is to find the largest number $\rho(s, \mu)$ such that for all $p \in P$

$$
\operatorname{Re}\left\{p(z)+s z p^{\prime}(z) /(p(z)+\mu)\right\}>0, \quad|z|<\rho(s, \mu),
$$

holds, where $-1 \neq \mu \in \mathbf{C}, s>0$. We shall use this result to solve a problem posed by Yoshikawa [9] and dealing with the 'radius of spiral-convexity' of spirallike functions in $\Delta$.

In the last section we shall derive the exact upper bounds of the functionals

Received by the editors October 14, 1975.

AMS (MOS) subject classifications (1970). Primary 30A76, 30A40; Secondary 30A38, 30A32.

Key words and phrases. Functions with positive real part, Hadamard product, spiral-convexity. 
$(\operatorname{Re} p(z)) /|p(z)+i \tan \alpha|$ and $\left|z p^{\prime}(z) /(p(z)+i \tan \alpha)\right|$ for $p \in P$. Crude bounds, in particular of the second entity, have been used by several authors [1], [3], [7], but a sharp result has not so far been obtained.

2. Let

$$
f(z)=\sum_{k=0}^{\infty} a_{k} z^{k}, \quad g(z)=\sum_{k=0}^{\infty} b_{k} z^{k}
$$

be analytic in $\Delta$. The function

$$
(f * g)(z):=\sum_{k=0}^{\infty} a_{k} b_{k} z^{k}
$$

is also analytic in $\Delta$ and is called the Hadamard product of $f$ and $g$.

Theorem 1. For $p \in P$ let

$$
\hat{p}(z):=\exp \left(\int_{0}^{z} \frac{p(\zeta)-1}{\zeta} d \zeta\right) .
$$

Let $F(z)$ be analytic in $\Delta, F(0) \neq 0$, and let $t \geqslant 0$. Then we have for fixed $z_{0} \in \Delta:$

$$
\min _{p \in P} \operatorname{Re} \frac{\left(F *\left(p \hat{p}^{t}\right)\right)\left(z_{0}\right)}{\left(F * \hat{p}^{t}\right)\left(z_{0}\right)}=\min _{p \in P_{0}} \operatorname{Re} \frac{\left(F *\left(p \hat{p}^{t}\right)\right)\left(z_{0}\right)}{\left(F * \hat{p}^{t}\right)\left(z_{0}\right)},
$$

whenever the right-hand side of (3) is nonnegative.

It should be pointed out that

$$
t \frac{\left(F *\left(p \hat{p}^{t}\right)\right)(z)}{\left(F * \hat{p}^{t}\right)(z)}=\frac{z g^{\prime}(z)}{g(z)}+t-1
$$

where $g(z)=z\left(F * \hat{p}^{t}\right)(z)$.

Theorem 1 is almost trivial for $t=0$. The case $t=1$, however, contains the truth of the Pólya-Schoenberg conjecture for convex univalent functions in $\Delta$.

REMARK 1. It is easy to see that the right-hand side of (3) is nonnegative if and only if

$$
F *(1-\varepsilon z) /(1-z)^{1+2 t} \neq 0, \quad|z|<\left|z_{0}\right|,|\varepsilon| \leqslant 1 .
$$

This relation is useful for applications of Theorem 1 .

Let $\alpha<1$. By $S_{\alpha}^{*}$ we denote the class of functions starlike of order $\alpha$ in $\Delta$, i.e. $f \in S_{\alpha}^{*}$ if and only if $f$ is analytic in $\Delta, f(0)=f^{\prime}(0)-1=0$, and fulfils $\operatorname{Re}\left(z f^{\prime}(z) / f(z)\right)>\alpha, z \in \Delta$. In particular we have

$$
s_{\alpha}:=z /(1-z)^{2-2 \alpha} \in S_{\alpha}^{*} \text {. }
$$

By $s_{\alpha}^{-1}$ we denote that well-defined analytic function which satisfies the relations

$$
s_{\alpha}^{-1} * s_{\alpha}=z /(1-z), \quad s_{\alpha}^{-1}(0)=0 .
$$

We shall require the following lemmata. 
Lemma 1 (Suffridge [7, Theorem 7]). For $\beta<1$ we have $f \in S_{\beta}^{*}$ if and only if $s_{\beta}^{-1} * f * g \in S_{\beta}^{*}$ for all $g \in S_{\beta}^{*}$.

Lemma 2 (Suffridge [7, Theorem 10]). Let $\alpha \leqslant \beta<1$. Then $f \in S_{\alpha}^{*}$ implies $s_{\alpha}^{-1} * s_{\beta} * f \in S_{\beta}^{*}$.

For $\alpha \leqslant \beta<1$ we introduce the set $V_{\alpha \beta}$ of analytic functions $h(z)$ in $\Delta$, $h(0)=0$, such that $h * f \in S_{\beta}^{*}$ for each $f \in S_{\alpha}^{*}$.

Lemma 3. Let $h(z)$ be analytic in $\Delta, h(0)=0$. Then $h \in V_{\alpha \beta}$ if and only if $h * s_{\alpha} \in S_{\beta}^{*}$.

Proof. Let $g \in S_{\beta}^{*}, h:=s_{\alpha}^{-1} * g$. Then for each $f \in S_{\alpha}^{*}$,

$$
f * h=\left(s_{\alpha}^{-1} * s_{\beta} * f\right) * s_{\beta}^{-1} * g \in S_{\beta}^{*}
$$

by Lemma 1 , since $s_{\alpha}^{-1} * s_{\beta} * f \in S_{\beta}^{*}$ by Lemma 2 . On the other hand, $h \in V_{\alpha \beta}$ implies $h * s_{\alpha} \in S_{\beta}^{*}$ since $s_{\alpha} \in S_{\alpha}^{*}$.

Proof of Theorem 1. Lemma 3 shows that for $\alpha \leqslant \beta<1$ and a certain $h(z)$ analytic in $\Delta, h(0)=0$, we have

$$
\operatorname{Re} \frac{z(h * g)^{\prime}(z)}{(h * g)(z)}>\beta \quad \text { for all } g \in S_{\alpha}^{*}, z \in \Delta,
$$

if and only if

$$
\operatorname{Re} \frac{z\left(h * s_{\alpha}\right)^{\prime}(z)}{\left(h * s_{\alpha}\right)(z)}>\beta, \quad z \in \Delta .
$$

Now let $p \in P, t>0$. Obviously $z \hat{p}^{t}(z) \in S_{1-t}^{*}$ and, in particular, for $p_{0}(z)$ $=(1+z) /(1-z)$ we have $z \hat{p}_{0}^{t}(z)=s_{1-t}(z)$. In this notation, (5) and (6) $\operatorname{read}(1-t \leqslant \beta<1)$ :

$$
\operatorname{Re} t \frac{\left(h *\left(z p \hat{p}^{t}\right)\right)(z)}{\left(h * z \hat{p}^{t}\right)(z)}>\beta+t-1 \text { for all } p \in P, z \in \Delta,
$$

if and only if

$$
\operatorname{Re} t \frac{\left(h *\left(z p_{0} \hat{p}_{0}^{t}\right)\right)(z)}{\left(h * z \hat{p}_{0}^{t}\right)(z)}>\beta+t-1, \quad z \in \Delta .
$$

Now assume

$$
\min _{p \in P_{0}} \operatorname{Re} \frac{\left(F * p \hat{p}^{t}\right)\left(z_{0}\right)}{\left(F * \hat{p}^{t}\right)\left(z_{0}\right)}=v \geqslant 0
$$

and put $h(z):=z F\left(z_{0} z\right), v=(\beta+t-1) / t$. Thus (9) implies (8) and, hence, (7), which in turn may be written as

$$
\min _{p \in P} \operatorname{Re} \frac{\left(F * p \hat{p}^{t}\right)\left(z_{0}\right)}{\left(F * \hat{p}^{t}\right)\left(z_{0}\right)}=v .
$$

The case $t=0$ is well known and follows from the Herglotz representation of the functions in $P$. 
3. In this section we give a simple application of Theorem 1. For -1 $\neq \mu \in \mathbf{C}, s>0$, let

$$
F(z):=\left(\frac{\mu+1}{s}-1\right) \frac{1}{1-z}+\frac{1}{(1-z)^{2}} .
$$

Then for $p \in P$ we obtain

$$
\frac{\left(F *\left(p \hat{p}^{1 / s}\right)\right)(z)}{\left(F * \hat{p}^{1 / s}\right)(z)}=p(z)+s \frac{z p^{\prime}(z)}{p(z)+\mu} .
$$

Corollary 1. For $p \in P$ and

$$
|z|<\frac{|\mu+1|}{\left[A+\left(A^{2}-\left|\mu^{2}-1\right|^{2}\right)^{1 / 2}\right]^{1 / 2}}, \quad A=2(s+1)^{2}+|\mu|^{2}-1,
$$

we have

$$
\operatorname{Re}\left(p(z)+s \frac{z p^{\prime}(z)}{p(z)+\mu}\right)>0 .
$$

The bound given by (12) is best possible.

Proof. From Remark 1 we deduce that we only have to find the largest radius $\rho=\rho(s, \mu)$ such that for $F$, given by (11),

$$
F * \frac{1-\varepsilon z}{(1-z)^{1+2 / s}} \neq 0, \quad|z|<\rho(s, \mu),|\varepsilon| \leqslant 1 .
$$

This is equivalent to the condition

$$
-s \frac{\varepsilon z}{1-\varepsilon z}+(s+2) \frac{z}{1-z} \neq-\mu-1, \quad|z|<\rho(s, \mu),|\varepsilon| \leqslant 1,
$$

and thus with $\left|2 \rho^{2}+(\mu+1)\left(1-\rho^{2}\right)\right| \geqslant 2 \rho(s+1)$. This leads immediately to (12).

It should be mentioned that one can use Theorem 1 to compute

$$
m(r)=\min _{p \in P} \min _{|z| \leqslant r} \operatorname{Re}\left(p(z)+\frac{s z p^{\prime}(z)}{p(z)+\mu}\right)
$$

as long as $m(r)$ is nonnegative.

Let $f(z)$ be analytic in $\Delta, f(0)=f^{\prime}(0)-1=0$. Yoshikawa [9] called $f(z)$ " $\gamma$-spiral-convex" if it satisfies the condition

$$
\operatorname{Re}\left(1+\frac{z f^{\prime \prime}(z)}{f^{\prime}(z)}+i \tan \gamma \frac{z f^{\prime}(z)}{f(z)}\right)>0
$$

in $\Delta$ and gave a geometrical interpretation of this condition. He proved that every $\gamma$-spiral-convex function is $\gamma$-spirallike, i.e. it fulfils

$$
\operatorname{Re} e^{i \gamma} \frac{z f^{\prime}(z)}{f(z)}>0, \quad z \in \Delta .
$$

He posed the problem to find the largest radius $\rho=\rho(\gamma)$, such that every $\gamma$-spirallike function is $\gamma$-spiral-convex in $|z|<\rho(\gamma)$, thereby generalizing the 
radius of (0-spiral-)convexity of the starlike ( $=0$-spirallike) functions in $\Delta$.

It is easily seen that this problem is equivalent to the following question: What is the greatest number $\rho(\gamma)$ such that

$$
\operatorname{Re}\left(p(z)+\frac{z p^{\prime}(z)}{p(z)+i \tan \gamma}\right)>0, \quad|z|<\rho(\gamma),
$$

for all $p \in P$. This question has been answered in Corollary 1 and thus we can state

THEOREM 2. The greatest common radius $\rho(\gamma)$ of $\gamma$-spiral-convexity of $\gamma$ spirallike functions in $\Delta$ is given by

$$
\rho(\gamma)=\sqrt{1+3 \cos ^{2} \gamma}-\sqrt{3} \cos \gamma .
$$

Yoshikawa himself was able to show that the value given in (16) is an upper bound for $\rho(\gamma)$.

4. In this last section we derive upper bounds of two special functionals on $P$. The method used is completely apart from those discussed in the previous chapters. The extremal functions, however, belong to $P_{0}$ too.

TheOREM 3. For $p \in P$ and $\alpha \in(-\pi / 2, \pi / 2)$ the estimate

$$
\frac{\operatorname{Re} p(z)}{|p(z)+i \tan \alpha|} \leqslant \begin{cases}\frac{\left(1-|z|^{2}\right) \cos \alpha}{1-2|z||\sin \alpha|+|z|^{2}}, & |z|<\left|\tan \frac{\alpha}{2}\right|, \\ 1, & |z| \geqslant\left|\tan -\frac{\alpha}{2}\right|,\end{cases}
$$

is valid and sharp. Equality holds for certain functions in $P_{0}$.

The estimate $\left|p^{\prime}(z)\right| \leqslant(2 \operatorname{Re} p(z)) /\left(1-|z|^{2}\right)$ holds for every $p \in P$ and $z \in \Delta$ (see[3]). Equality holds only for every $p \in P_{0}$ and $z \in \Delta$. Thus Theorem 3 has the following corollary.

Corollary 2. For $p \in P$ and $\alpha \in(-\pi / 2, \pi / 2)$ the estimate

$$
\left|\frac{z p^{\prime}(z)}{p(z)+i \tan \alpha}\right| \leqslant \begin{cases}\frac{2|z| \cos \alpha}{1-2|z||\sin \alpha|+|z|^{2}}, & |z|<\left|\tan \frac{\alpha}{2}\right|, \\ \frac{2|z|}{1-|z|^{2}}, & |z| \geqslant\left|\tan \frac{\alpha}{2}\right|,\end{cases}
$$

is valid and sharp. Equality holds only for certain functions $p \in P_{0}$.

We shall require the following well-known fact:

The range of the functional $w=p(z), z$ fixed, over $P$ is the closed disc $K_{|z|}$ with center $\left(1+|z|^{2}\right) /\left(1-|z|^{2}\right)$ and radius $2|z| /\left(1-|z|^{2}\right)$. Furthermore, the points on $\partial K_{|z|}$ correspond only to functions in $P_{0}$.

$$
\frac{\operatorname{Re} p(z)}{|p(z)+i \tan \alpha|} \leqslant \frac{\operatorname{Re} p(z)}{\operatorname{Re} p(z)}=1,
$$

and equality can hold if and only if there exists a $w_{1} \in \partial K_{|z|}$ with $\operatorname{Im} w_{1}=$ $-\tan \alpha$. This is the case if and only if $|z| \geqslant|\tan (\alpha / 2)|$ and equality holds for a certain $p \in P_{0}$. This proves the bottom half of (17).

Now let $z_{0} \in \Delta$ be fixed, $\left|z_{0}\right|<|\tan (\alpha / 2)|$. Let $p_{0} \in P$ be an extremal 
function for our functional at $z_{0}$ ( $P$ is a normal family). The function

$$
p^{*}(z)=\left(p_{0}(z)+i \lambda\right) /\left(1+i \lambda p_{0}(z)\right)
$$

belongs to $P$ for arbitrary $\lambda \in \mathbf{R}$ and gives the variational formula

$$
p^{*}(z)=p_{0}(z)+i \lambda\left(1-p_{0}^{2}(z)\right)+o(\lambda) .
$$

Using the extremal property of $p_{0}$ and this variation we are led to the condition $\cos \alpha\left|p_{0}(z)+i \tan \alpha\right|=1$. If we let $p_{0}\left(z_{0}\right)+i \tan \alpha=\sec \alpha e^{i \gamma}$ it follows for $p \in P$

$$
\frac{\operatorname{Re} p\left(z_{0}\right)}{\left|p\left(z_{0}\right)+i \tan \alpha\right|} \leqslant \frac{\operatorname{Re} p_{0}\left(z_{0}\right)}{\left|p_{0}\left(z_{0}\right)+i \tan \alpha\right|}=\cos \gamma,
$$

and we are left with the problem to maximize $\cos \gamma$ within the set of points $\sec \alpha e^{i \gamma}-i \tan \alpha \in K_{\left|z_{0}\right|}$. It is easily seen that the extremal value is

$$
\frac{\left(1-\left|z_{0}\right|^{2}\right) \cos \alpha}{1-2\left|z_{0}\right||\sin \alpha|+\left|z_{0}\right|^{2}}
$$

and corresponds to a boundary point of $K_{\left|z_{0}\right|}$. The theorem is proven.

ADDED IN PROOF. Theorem 2 has also been established by P. Eenigenburg and H. Yoshikawa in their paper An application of the method of Zmorovič in geometric function theory, J. Math. Anal. Appl. (to appear).

\section{REFERENCES}

1. S. D. Bernardi, New distortion theorems for functions of positive real part and applications to the partial sums of univalent convex functions, Proc. Amer. Math. Soc. 45 (1974), 113-118. MR 50 \# 10223.

2. M. S. Robertson, Variational methods for functions with positive real part, Trans. Amer. Math. Soc. 102 (1962), 82-93. MR 24 \# A3288.

3. Extremal problems for analytic functions with positive real part and applications, Trans. Amer. Math. Soc. 106 (1963), 236-253. MR 26 \#325.

4. St. Ruscheweyh, New criteria for univalent functions, Proc. Amer. Math. Soc. 49 (1975), $109-115$.

5. St. Ruscheweyh and T. Sheil-Small, Hadamard products of schlicht functions and the Pólya-Schoenberg conjecture, Comment. Math. Helv. 48 (1973), 119-135. MR 48 \#6393.

6. K. Sakaguchi, $A$ variational method for functions with positive real part, J. Math. Soc. Japan 16 (1964), 287-297. MR 31 \#1375.

7. D. B. Shaffer, Distortion theorems for a special class of analytic functions, Notices Amer. Math. Soc. 19 (1972), A-519. Abstract \#72T-B152.

8. T. J. Suffridge, Starlike functions as limits of polynomials (preprint).

9. H. Yoshikawa, On a subclass of spiral-like functions, Mem. Fac. Sci. Kyushu Univ. Ser. A 25 (1971), 271-279. MR 46 \#9320.

10. V. A. Zmorovič, On bounds of convexity for starlike functions of order $\alpha$ in the circle $|z|<1$ and in the circular region $0<|z|<1$, Mat. Sb. 68 (110) (1965), 518-526; English transl., Amer. Math. Soc. Transl. (2) 80 (1969), 203-213. MR 33 \# 5875.

11. , On the bounds of starlikeness and univalence in certain classes of functions regular in the circle $|z|<1$, Ukrain. Mat. Ż. 18 (1966), 28-39; English transl., Amer. Math. Soc. Transl. (2) 80 (1969), 227-242. MR 33 \#7525.

Abteilung Mathematik, Universität Dortmund, 4600 Dortmund 50, West Germany

Department of Mathematics, Punjabi University, Patiala (Pb), India 